\title{
Effect of Heat Source/Sink on Free Convective Flow of a Polar Fluid between Vertical Concentric Annuli
}

\author{
Arun Kumar Singh, Ashok Kumar Singh \\ Department of Mathematics, Institute of Science, Banaras Hindu University, Varanasi, India \\ Email: arunsingh253@gmail.com, ashok@bhu.ac.in
}

How to cite this paper: Singh, A.K. and Singh, A.K. (2017) Effect of Heat Source/Sink on Free Convective Flow of a Polar Fluid between Vertical Concentric Annuli. Journal of Applied Mathematics and Physics, 5, 1750-1762.

https://doi.org/10.4236/jamp.2017.59148

Received: June 22, 2017

Accepted: September 17, 2017

Published: September 20, 2017

Copyright $\odot 2017$ by authors and Scientific Research Publishing Inc. This work is licensed under the Creative Commons Attribution International License (CC BY 4.0).

http://creativecommons.org/licenses/by/4.0/

\section{Open Access}

\begin{abstract}
The purpose of this paper is to find the effect of heat source/sink parameter on free convective flow of a polar fluid in open-ended vertical concentric annuli. Exact solutions of the non-dimensional differential equations describing the flow model have been obtained one by one for two different cases of source and sink. To observe the effect of the physical parameters such as source/sink and vertex viscosity, the numerical results of the velocity and microrotational velocity are finally shown on the graphs.
\end{abstract}

\section{Keywords}

Polar Fluid, Free Convection, Isothermal and Constant Heat Flux Cases, Temperature Dependent Heat Source/Sink, Vertical Annuli

\section{Introduction}

Free convection occurs due to the impact of gravity on the heated fluid of variable density and thus relative buoyancy of the fluid. It occurs at small velocities in the presence of large temperature difference. Example of the free convection is a pot of boiling water in which the hot and less-dense water on the bottom layer moves upwards in plumes while the cool and denser water near the top of a pot likewise sinks. The fully developed laminar free convective flow of a fluid heated asymmetrically between two vertical plates has been described very well by Aung [1]. Nelson and Wood [2] have obtained the solution in the case of the combined influence of heat and mass transfer on natural convection in vertical channels.

The heat transfer phenomena in non-Newtonian fluid flows through ducts are found in analysis of the polymer in chemical engineering and applications of 
fluid film lubrication etc. The behaviors and theories of many non-Newtonian fluids have been proposed by many researchers. The concepts of such fluids demand a new idea which differs from the real fluids. Here, we shall study a special family of non-Newtonian fluid such as polar fluid, whose substantial equations with couple stresses have been described by Aero et al. [3], D'ep [4] and Cowin [5]. Some examples of a polar fluid are mud, crude oil, body fluids, lubricants with polymer additives etc. Comparative studies of the Poiseuille flow corresponding to a polar fluid under various boundary conditions with applications to blood flow have been studied by Chaturani and Biswas [6]. Singh et al. [7] have studied the natural convection under a radial magnetic field in vertical concentric annuli. Using the generalized Forchheimer's model, Raptis and Takhar [8] have studied the flow of a polar fluid in a porous medium bounded by an infinite plate. Ogulu [9] has obtained the analytical solutions for the problem concerned with free convective flow of a polar fluid past a vertical porous plate with couple stresses and radiation, where the thermal energy of the plate is assumed to oscillate about a mean value. Later, the studies of flow formation in different geometries with and without heat source have been attempted by Cheng [10] [11]. Patil [12] has obtained analytic solution for the problem of free convection on the oscillatory flow of a polar fluid within a porous medium with variable heat flux.

Fluid flow with internal heat sources/sinks finds physical as well as theoretical interest. The fluid motion develops slowly following the development of non-uniformity in the temperature field. However, when the thermal energy differences are admirably large, the volumetric heat generation or absorption term exerts strong impact on the heat transfer and as a consequence on the fluid flow as well. The natural convective flows with heat generation from an isothermal horizontal circular cylinder have been studied by Molla et al. [13]. The effects of chemical reaction on free convective flow of a polar fluid in a porous medium with internal heat generation have been described by Patil and Kulkarni [14]. The natural convective flows of a polar fluid between vertical concentric annuli have been examined by Ravi and Singh [15]. Mohamed et al. [16] have obtained the solutions for thermal radiation and magnetic effects on free convective flow of a polar fluid in a porous medium with internal heat generation and chemical reaction. Moreover, Ravi et al. [17] have presented the free convection of a micropolar fluid between two vertical walls by taking into account the temperature dependent source/sink. Perdikis and Rapti [18] have studied the flow of an incompressible and electrically conducting polar fluid in the presence of a magnetic field. Motivated by the above papers, a boundary value problem is considered here to investigate analytically the effect of heat source/sink on the velocity and microrotational velocity when outer cylinder is isothermal while thermal boundary condition at the inner one is mixed kind. Analytical solutions obtained here are compared to the solution of natural convection of a polar fluid in vertical concentric annuli by Ravi and Singh [15] to check the accuracy of the solution. Finally, the effects of heat source or sink, vertex viscosity and radii ratio 
parameters have been shown by graphs.

\section{Mathematical Formulations}

We have considered steady and laminar free convective flow of a polar fluid inside vertical concentric annulus of infinite length with temperature dependent heat source/sink. The $z^{\prime}$-axis is taken along the axis of cylinder in the vertical upward direction and $r^{\prime}$-axis is in the radial direction. Let $a$ and $b$ be the radii of inner and outer cylinders respectively. In this case the inner cylinder is cooled or heated either at a constant heat flux or isothermally so that its temperature (i.e. temperature of outer surface of the inner cylinder) is different from the ambient temperature $T_{\infty}^{\prime}$. Since cylinders are of infinite length, the flow depends only on $r^{\prime}$. Using the Boussinesq approximation, the governing equation for free convective flow of a polar fluid with temperature dependent heat source or sink can be obtained as follows:

$$
\begin{gathered}
\left(v+\frac{k}{\rho}\right) \frac{1}{r^{\prime}} \frac{\mathrm{d}}{\mathrm{d} r^{\prime}}\left(r^{\prime} \frac{\mathrm{d} u^{\prime}}{\mathrm{d} r^{\prime}}\right)+\frac{k}{\rho}\left(\frac{N^{\prime}}{r^{\prime}}+\frac{\mathrm{d} N^{\prime}}{\mathrm{d} r^{\prime}}\right)+g \beta\left(T^{\prime}-T_{\infty}^{\prime}\right)=0, \\
\frac{\mathrm{d}}{\mathrm{d} r^{\prime}}\left(r^{\prime} \frac{\mathrm{d} N^{\prime}}{\mathrm{d} r^{\prime}}\right)-\frac{N^{\prime}}{r^{\prime}}=0, \\
\frac{1}{r^{\prime}} \frac{\mathrm{d}}{\mathrm{d} r^{\prime}}\left(r^{\prime} \frac{\mathrm{d} T^{\prime}}{\mathrm{d} r^{\prime}}\right)+\frac{Q_{0}}{\rho C_{p}}=0 .
\end{gathered}
$$

The boundary conditions for the model are as follows:

$$
\begin{array}{ll}
u^{\prime}=0, N^{\prime}=-\frac{1}{2} \frac{\mathrm{d} u^{\prime}}{\mathrm{d} r^{\prime}}, T^{\prime}=T_{w}^{\prime} \text { or } \frac{\mathrm{d} T^{\prime}}{\mathrm{d} r^{\prime}}=-\frac{q}{k}, & \text { at } r^{\prime}=a, \\
u^{\prime}=0, N^{\prime}=0, T^{\prime}=T_{\infty}^{\prime} & \text { at } r^{\prime}=b .
\end{array}
$$

For the non-dimensionalization of governing Equations (1) - (3) and boundary conditions given by (4), we use the non-dimensional variables and parameters for the considered flow, defined as follows:

$$
\begin{aligned}
& u=\frac{u^{\prime} v}{\left(\beta g a^{2} \Delta T\right)}, N=\frac{N^{\prime} v}{(\beta g a \Delta T)}, S=\frac{Q_{0}}{\rho C_{p}}, \\
& T=\frac{T^{\prime}-T_{\infty}^{\prime}}{\Delta T}, \lambda=\frac{b}{a}, r=\frac{r^{\prime}}{a}, R=\frac{k}{\mu} .
\end{aligned}
$$

In this equation, $\Delta T$ is $\left(T_{w}^{\prime}-T_{\infty}^{\prime}\right)$ or $\frac{q^{\prime} a}{k}$ according as inner cylinder is maintained at constant temperature $T_{w}^{\prime}$ or constant heat flux $q^{\prime}$. Use of Equation (5) into Equations (1) - (3) has produced the following non-dimensional equations:

$$
\begin{gathered}
(1+R)\left[\frac{\mathrm{d}^{2} u}{\mathrm{~d} r^{2}}+\frac{1}{r} \frac{\mathrm{d} u}{\mathrm{~d} r}\right]+R\left[\frac{N}{r}+\frac{\mathrm{d} N}{\mathrm{~d} r}\right]+T=0, \\
r \frac{\mathrm{d}^{2} N}{\mathrm{~d} r^{2}}+\frac{\mathrm{d} N}{\mathrm{~d} r}-\frac{N}{r}=0,
\end{gathered}
$$




$$
\frac{\mathrm{d}^{2} T}{\mathrm{~d} r^{2}}+\frac{1}{r} \frac{\mathrm{d} T}{\mathrm{~d} r}+S=0 .
$$

Further, the corresponding boundary conditions in dimensionless form are obtained as

$$
\begin{array}{ll}
u=0, N=-\frac{1}{2} \frac{\mathrm{d} u}{\mathrm{~d} r}, T=1 \text { or } \frac{\mathrm{d} T}{\mathrm{~d} r}=-1, & \text { at } r=1, \\
u=0, N=0, T=0, & \text { at } r=\lambda .
\end{array}
$$

The boundary conditions for the temperature field at the inner cylinder are of the Dirichlet and Newmann types. For a unified solution for both boundary conditions, these boundary conditions for temperature field at the inner cylinder can be combined into a single boundary condition known as boundary condition of third kind at the inner cylinder. By doing so, a combined condition is obtained as follows:

$$
A \frac{\mathrm{d} T}{\mathrm{~d} r}+B T=C \text {, at } r=1 .
$$

The corresponding cases can be obtained by assigning appropriate values to $A, B$ and $C$ in Equation (10). The solution of Equations (6) - (8) subject to their suitable boundary conditions are obtained as for the cases of source and sink respectively.

\section{Case 1 When source is present}

In this case, $S>0$ and as a result of which the solutions for $T, u$ and $N$ are obtained as follows:

$$
\begin{gathered}
T=A_{1} \log r+A_{2}-\frac{S r^{2}}{4}, \\
u=A_{22} \log r+A_{21}+\left(A_{7} A_{19}+A_{8}\right) r^{2}+A_{9} r^{2} \log r+A_{10} r^{4}, \\
N=A_{19} r+\frac{A_{20}}{r} .
\end{gathered}
$$

Using Equation (10), the dimensionless skin-frictions at outer surface of inner cylinder and inner surface of outer cylinder in the presence of source are derived as

$$
\begin{gathered}
\tau_{1}=\left(\frac{\mathrm{d} u}{\mathrm{~d} r}\right)_{r=1}=A_{22}+2\left(A_{7} A_{19}+A_{8}\right)+A_{9}+4 A_{10}, \\
\tau_{\lambda}=\left(\frac{\mathrm{d} u}{\mathrm{~d} r}\right)_{r=\lambda}=\frac{A_{22}}{\lambda}+2\left(A_{7} A_{19}+A_{8}\right) \lambda+A_{9} \lambda(2 \lambda \log \lambda+1)+4 A_{10} \lambda^{3} .
\end{gathered}
$$

\section{Case 2 When sink is present}

In the case of sink, $S<0$ and to find analytical solution, we take $S=-S i, S i>0$. Corresponding to this case, the solutions for $T, u$ and $N$ in terms of $S i$ are derived as follows:

$$
\begin{gathered}
T=q_{2} \log r+q_{3}+\frac{\operatorname{Sir}^{2}}{4}, \\
u=q_{22} \log r+q_{21}+\left(q_{8} q_{19}+q_{9}\right) r^{2}+q_{10} r^{2} \log r+q_{11} r^{4},
\end{gathered}
$$




$$
N=q_{19} r+\frac{q_{20}}{r} .
$$

In addition, the dimensionless skin-frictions at outer surface of inner cylinder and inner surface of outer cylinder with sink are given by

$$
\begin{gathered}
\tau_{1}=\left(\frac{\mathrm{d} u}{\mathrm{~d} r}\right)_{r=1}=q_{22}+2\left(q_{8} q_{19}+q_{9}\right)+q_{10}+4 q_{11}, \\
\tau_{\lambda}=\left(\frac{\mathrm{d} u}{\mathrm{~d} r}\right)_{r=\lambda}=\frac{q_{22}}{\lambda}+2\left(q_{8} q_{19}+q_{9}\right) \lambda+q_{10} \lambda(2 \lambda \log \lambda+1)+4 q_{11} \lambda^{3} .
\end{gathered}
$$

\section{Results and Discussion}

The impacts of physical parameters such as the source $(S) / \operatorname{sink}(S i)$, vertex viscosity $(R)$ and radii ratio $(\lambda)$ on the velocity $(u)$, microrotational velocity $(N)$ are analyzed by presenting the numerical calculation of the analytical solution for constant heat flux case and isothermal case on the graphs.

Graphical representation in Figures 1-3 shows the variation of the velocity profiles with source, vertex viscosity and gap between the cylinders for both cases such as constant heat flux and isothermal. It is clear from these figures that the velocity of the fluid increases when the source and gap between the cylinders increases for both cases. When $\lambda>2.5$, the velocity is greater for the constant heat flux case compared to the isothermal case while reverse phenomenon occurs when $\lambda<2.5$. In light of this effect, we obtain the critical value of $\lambda$ at which the velocity profiles are the same for both cases. The nature of the velocity of the fluid is almost the same for constant heat flux and isothermal cases when the critical value of $\lambda=2.5$. The critical values 2.5 and 2.18 of $\lambda$ corresponding to $S=0.1$ and 0.4 , clearly show that the effect of source parameter is to decrease it. As in the case above, the velocity of the fluid decreases with increasing vertex viscosity parameters for both cases.

Figures 4-6 presents the variation of the microrotational velocity profiles with the source, vertex viscosity and gap between the cylinders for both the cases of

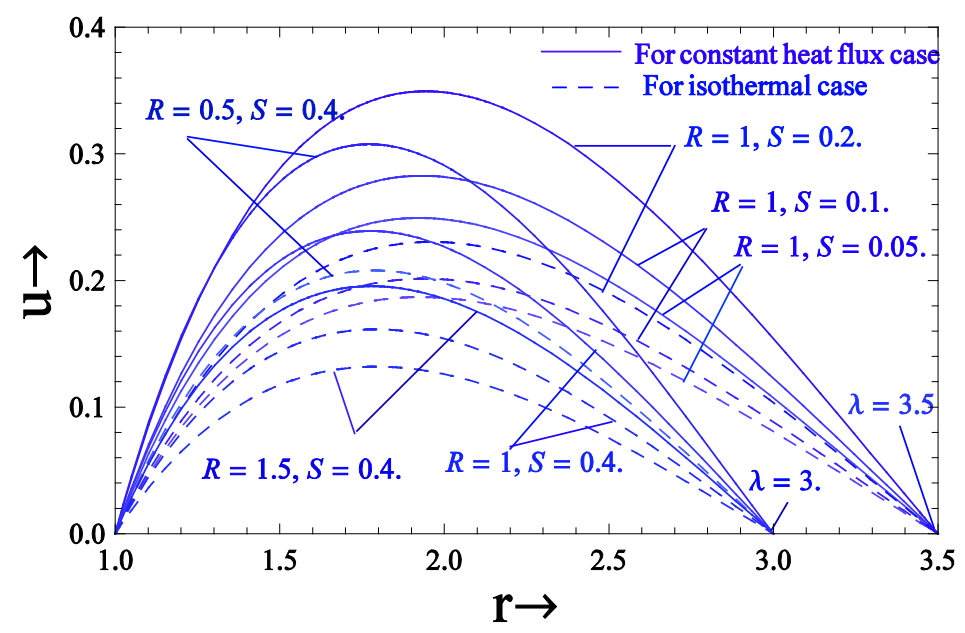

Figure 1. Velocity profiles for $\lambda>2.5$. 


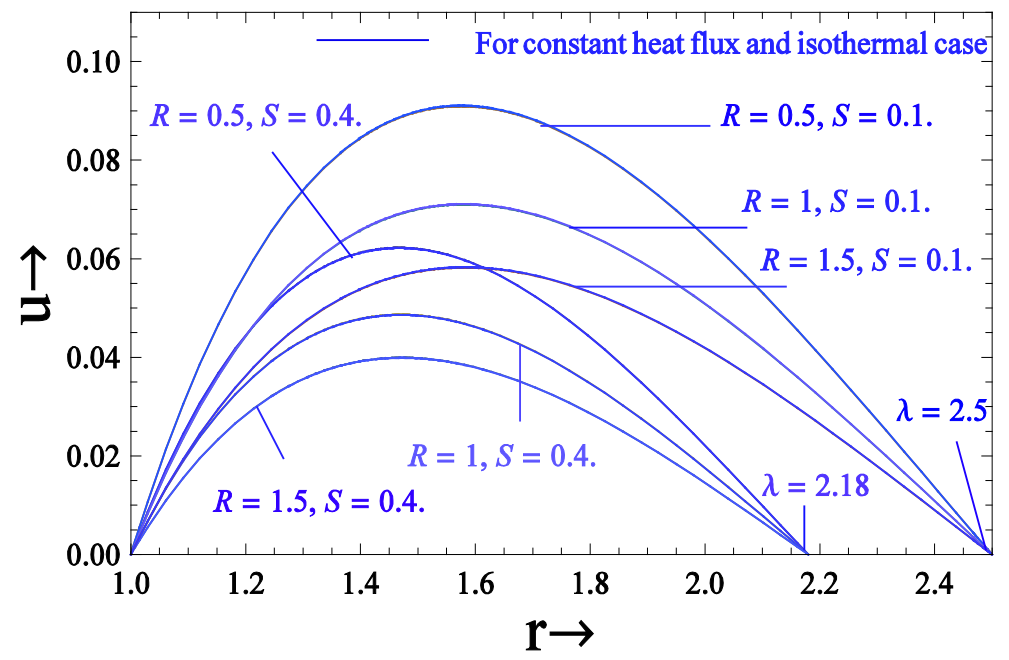

Figure 2. Velocity profiles for $\lambda(=2.5,2.18)$.

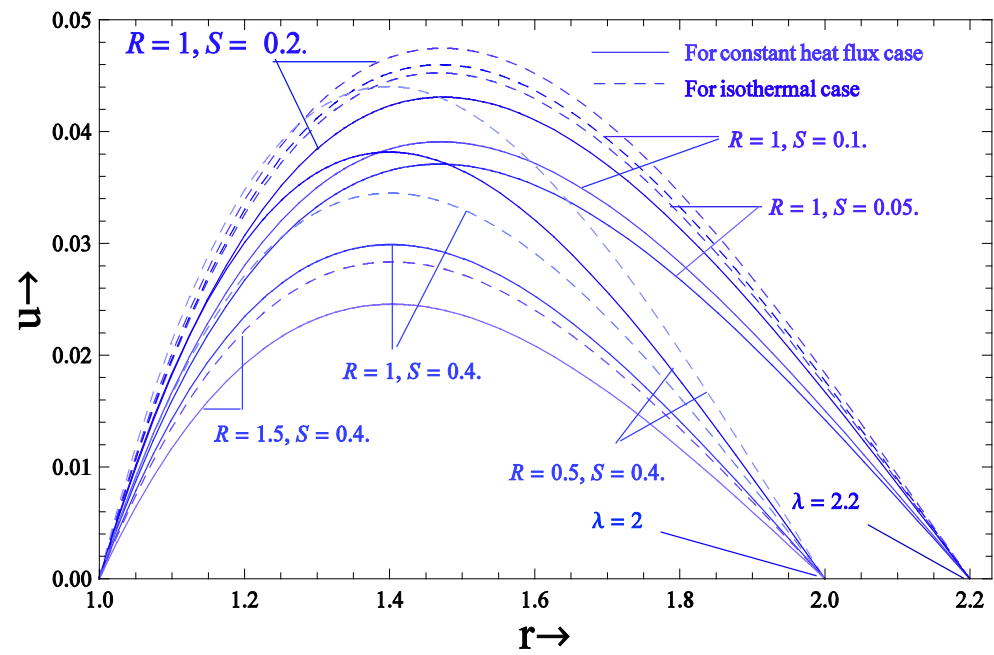

Figure 3. Velocity profiles for $\lambda<2.5$.

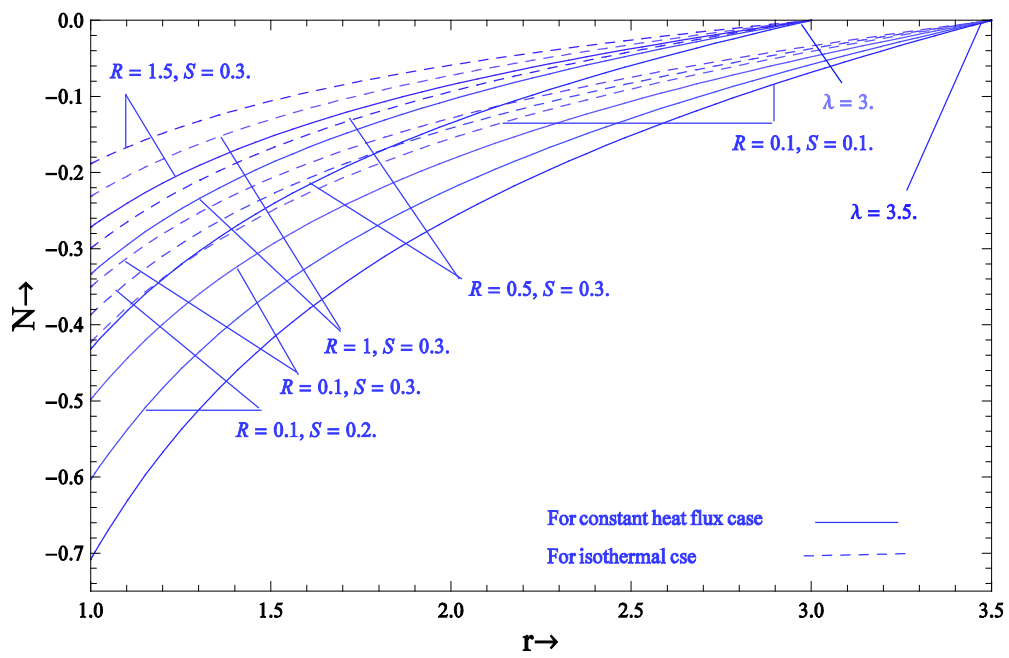

Figure 4. Influences of source and vertex viscosity parameters on microrotational velocity for $\lambda>2.5$. 


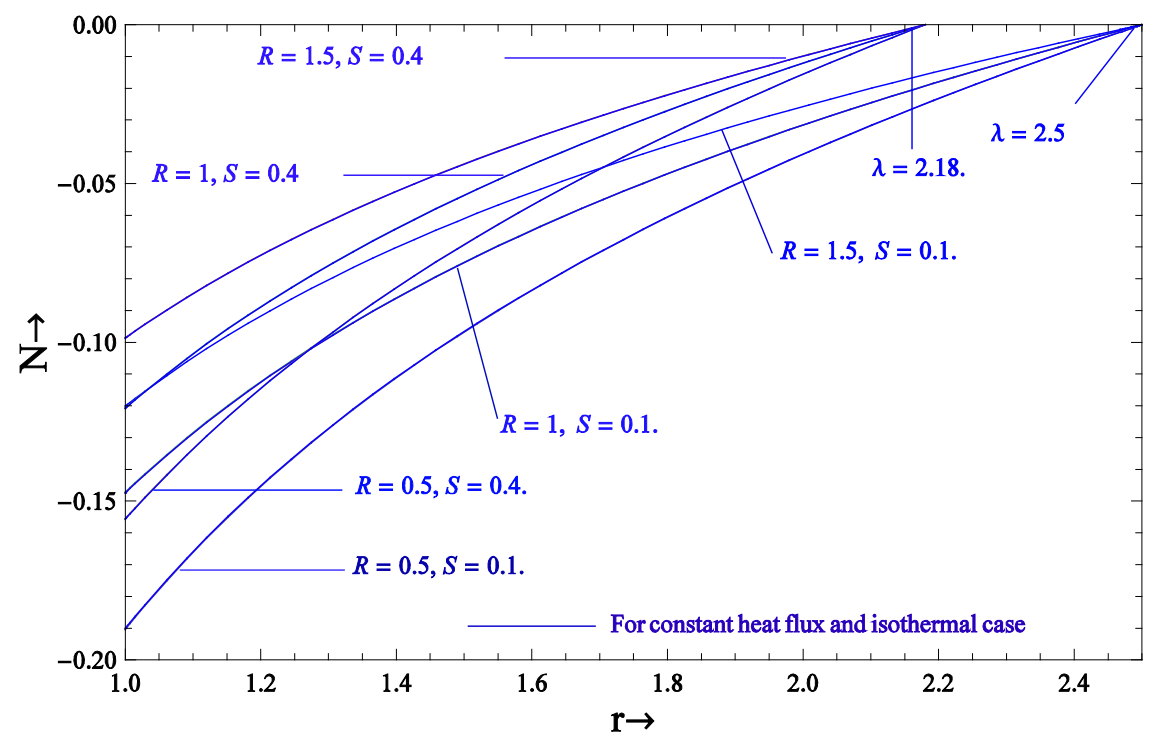

Figure 5. Effect of source $S(=0.1,0.4)$ and vertex viscosity $R(=0.5,1,1.5)$ parameters on microrotational velocity for $\lambda(=2.5,2.18)$.

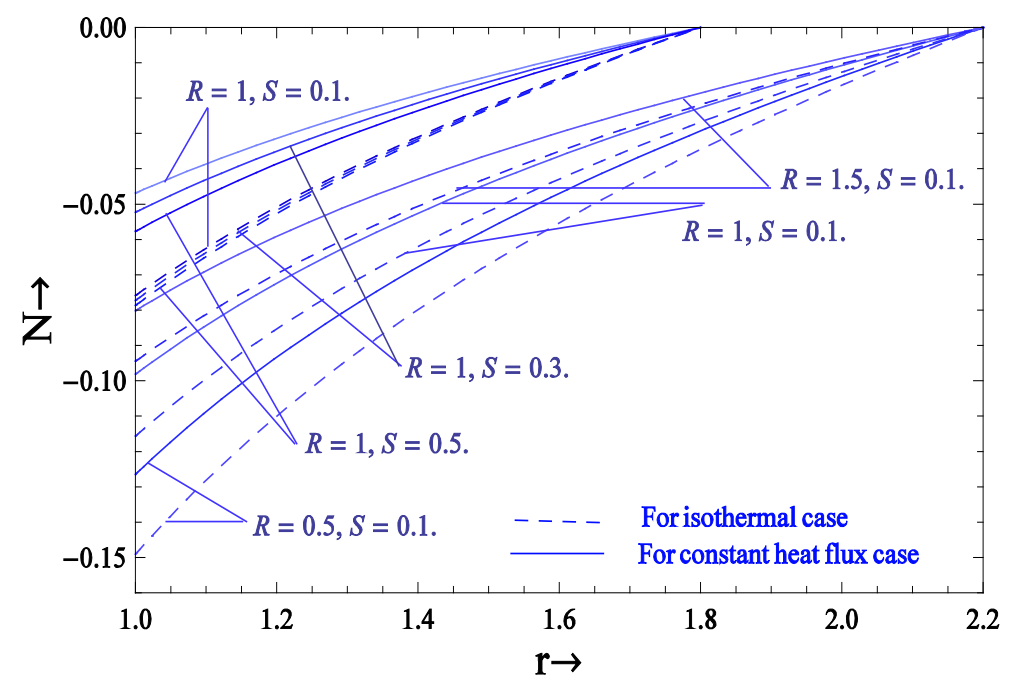

Figure 6. Effect of source and vertex viscosity parameter on microrotational velocity for $\lambda$ $<2.5$.

thermal heating. These figures demonstrate that the effect of the source parameters and gap between the cylinders on the microrotational velocity is to increases it for both cases. When $\lambda>2.5$, the microrotational velocity is more for the constant heat flux case compared to the isothermal case while reverse trend occurs when $\lambda<2.5$. Again, we reveal that the microrotational velocity profiles of a polar fluid for $\lambda=2.5$ are approximately the same for both cases and the critical values of $\lambda(=2.5,2.18)$ decrease with source $(S=0.1,0.4)$. The microrotational velocity of the fluid decreases with increasing vertex viscosity parameter for both types of thermal conditions. Figure 7 and Figure 8 describe that the velocity and microrotational velocity of a polar fluid with sink are the 
same for constant heat flux and isothermal cases when $\lambda(=2.9,3.26)$. In this case, we find that the critical value of $\lambda$ for the velocity and microrotational velocity of a polar fluid enhances with heat sink $\operatorname{Si}(=0.05,0.1)$ for constant heat flux and isothermal cases. As in the case above, the velocity and microrotational velocity of the fluid decreases with increasing vertex viscosity parameter for both cases.

The numerical values of skin-friction $\tau_{1}$ and $\tau_{\lambda}$ for isothermal and constant heat flux cases with temperature dependent heat source/sink are given in Table 1 . This table clearly reveals that the skin-friction on inner cylinder increases when heat source increases and decreases with increasing heat sink for

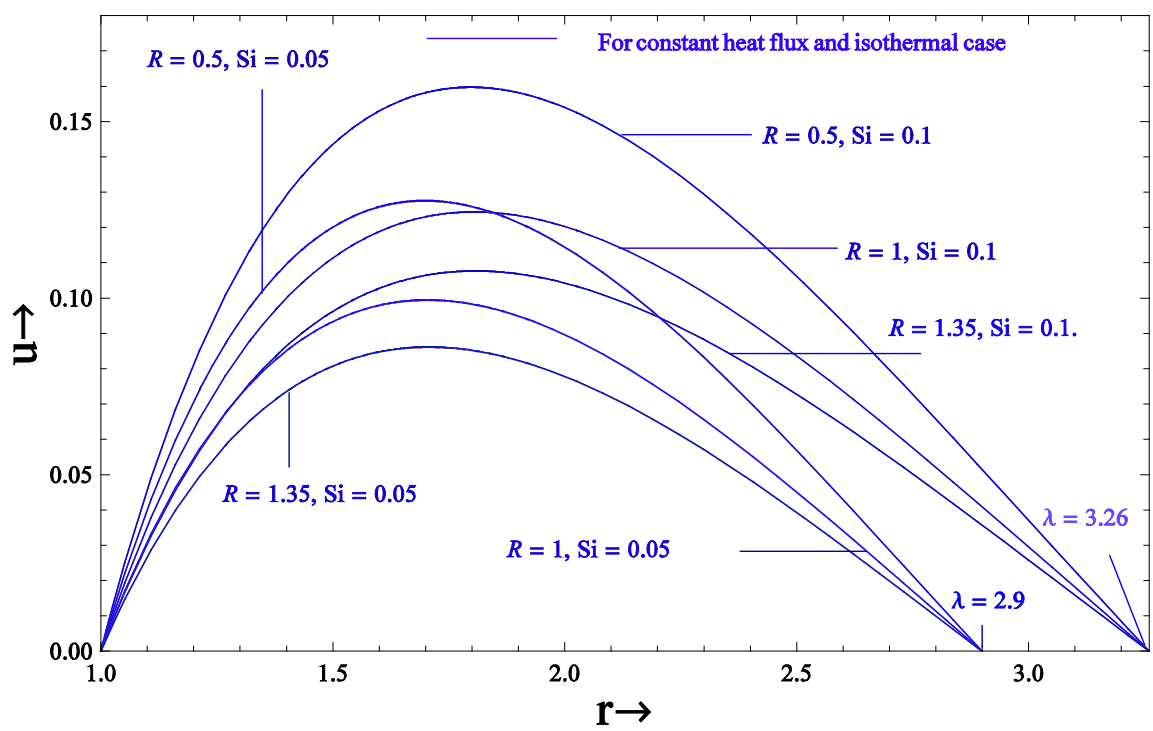

Figure 7. Velocity profiles for $\lambda(=2.9,3.26)$.

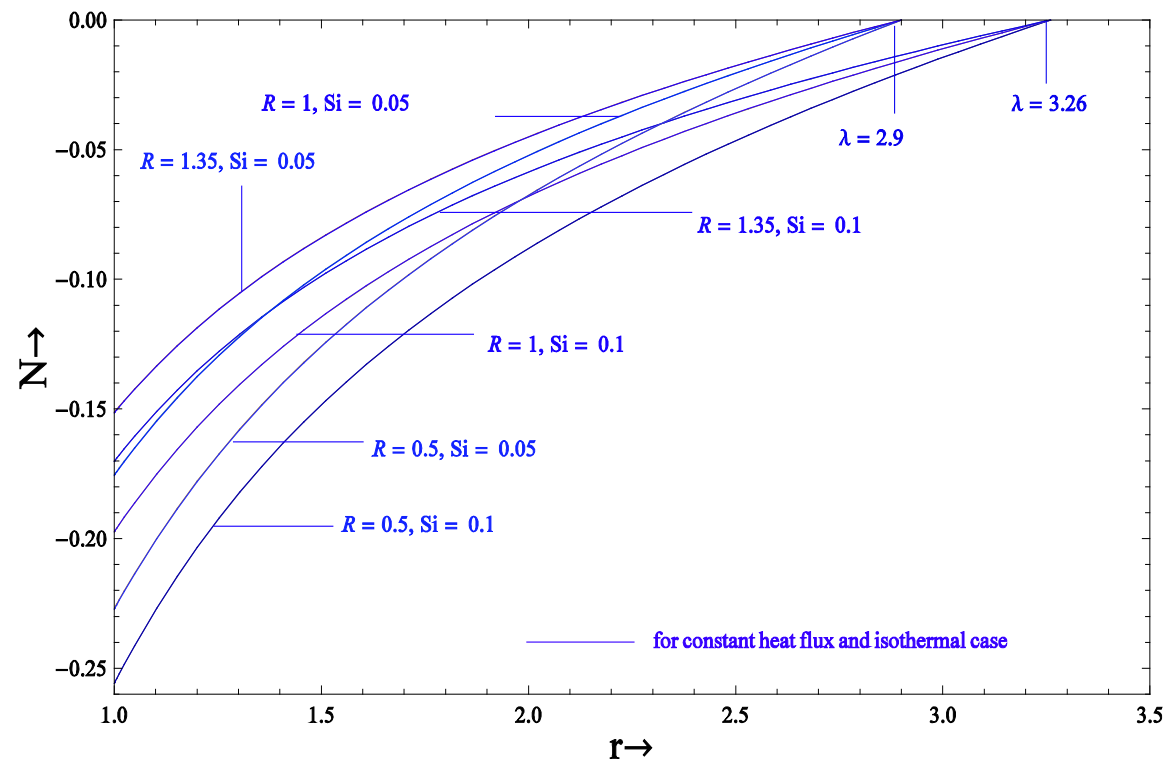

Figure 8. Impact of sink and vertex viscosity parameters on microrotational velocity for $\lambda(=2.9,3.26)$. 
Table 1. Numerical values of skin-friction for Isothermal and constant heat flux cases.

\begin{tabular}{|c|c|c|c|c|c|c|}
\hline$\lambda$ & $R$ & $S$ & $\tau_{1}$ & $\tau_{\lambda}$ & $\tau_{1}$ & $\tau_{\lambda}$ \\
\hline & & & \multicolumn{2}{|c|}{ Isothermal case } & \multicolumn{2}{|c|}{ Constant heat flux case } \\
\hline \multirow[t]{3}{*}{2} & 0.5 & 0.4 & 0.256830 & 0.362874 & 0.221607 & 0.279434 \\
\hline & 1 & 0.4 & 0.199520 & 0.266716 & 0.172157 & 0.204882 \\
\hline & 1.5 & 0.4 & 0.163120 & 0.210609 & 0.140749 & 0.161521 \\
\hline \multirow[t]{3}{*}{2.2} & 1 & 0.05 & 0.228733 & 0.540569 & 0.187285 & 0.435377 \\
\hline & & 0.1 & 0.231288 & 0.507115 & 0.196238 & 0.418162 \\
\hline & & 0.2 & 0.236334 & 0.440208 & 0.214145 & 0.383731 \\
\hline \multirow[t]{3}{*}{2.18} & 0.5 & 0.4 & 0.311553 & 0.412703 & 0.311181 & 0.411725 \\
\hline & 1 & 0.4 & 0.241724 & 0.303368 & 0.241435 & 0.302642 \\
\hline & 1.5 & 0.4 & 0.197466 & 0.239571 & 0.197230 & 0.238994 \\
\hline \multirow[t]{3}{*}{2.5} & 0.5 & 0.1 & 0.380305 & 0.950761 & 0.380938 & 0.952701 \\
\hline & 1 & 0.1 & 0.294494 & 0.706343 & 0.294985 & 0.707786 \\
\hline & 1.5 & 0.1 & 0.240279 & 0.561674 & 0.240679 & 0.562823 \\
\hline \multirow[t]{3}{*}{3} & 0.5 & 0.4 & 0.634018 & 0.161873 & 0.970214 & 1.413830 \\
\hline & 1 & 0.4 & 0.489771 & 0.111760 & 0.749479 & 1.045610 \\
\hline & 1.5 & 0.4 & 0.398995 & 0.0845528 & 0.610568 & 0.829060 \\
\hline \multirow[t]{3}{*}{3.5} & 1 & 0.05 & 0.512094 & 0.718880 & 0.687341 & 2.458200 \\
\hline & & 0.1 & 0.540174 & 1.395840 & 0.768326 & 2.358350 \\
\hline & & 0.2 & 0.596334 & 0.749748 & 0.930297 & 2.158650 \\
\hline \multirow[t]{2}{*}{2.6} & 1 & -0.1 & 0.290577 & 1.091910 & 0.247871 & 0.960497 \\
\hline & & -0.3 & 0.264736 & 1.407950 & 0.163622 & 1.096820 \\
\hline \multirow[t]{2}{*}{3} & 1 & -0.1 & 0.355741 & 1.691510 & 0.337969 & 1.627600 \\
\hline & & -0.2 & 0.328935 & 2.007460 & 0.255667 & 1.744000 \\
\hline \multirow[t]{3}{*}{2.9} & 0.5 & -0.05 & 0.454552 & 1.865410 & 0.453944 & 1.863230 \\
\hline & 1 & -0.05 & 0.351289 & 1.391350 & 0.350819 & 1.390320 \\
\hline & 1.35 & -0.05 & 0.303091 & 1.181810 & 0.302686 & 1.180430 \\
\hline \multirow[t]{3}{*}{3.26} & 0.5 & -0.1 & 0.511594 & 2.906310 & 0.511664 & 2.906590 \\
\hline & 1 & -0.1 & 0.394789 & 2.172470 & 0.394842 & 2.172680 \\
\hline & 1.35 & -0.1 & 0.340387 & 1.846040 & 0.340340 & 1.846220 \\
\hline \multirow[t]{2}{*}{3.5} & 0.5 & -0.05 & 0.591340 & 3.163820 & 0.681396 & 3.556000 \\
\hline & & -0.1 & 0.554921 & 3.593900 & 0.576359 & 3.687260 \\
\hline \multirow[t]{2}{*}{4} & 0.5 & -0.05 & 0.697280 & 4.576040 & 0.875763 & 5.461230 \\
\hline & & -0.1 & 0.630025 & 5.360310 & 0.691653 & 5.665960 \\
\hline
\end{tabular}

isothermal and constant heat flux cases while reverse tendency occurs on outer cylinder. The skin-friction on both the cylinders increases with increasing values of $\lambda$ (gap between the inner and outer cylinder) for both cases. In Table 1, the 
comparative study of isothermal and constant heat flux cases shows that the skin-friction $\tau_{1}$ and $\tau_{\lambda}$ are greater for isothermal heating of the inner cylinder than constant heat flux case when the gap between cylinders are less than $\lambda=2.5$ and 3.26 times the radius of the inner cylinder with heat source/sink, while the effect is just reverse when the gap between the cylinders are greater than $\lambda=2.5$ and 3.26 times the radius of inner cylinder respectively. The numerical values of skin-frictions on both cylinders are almost same at $\lambda=2.5$ and 3.26 when $S=0.1$ and -0.1 .

\section{Conclusions}

The analytic solutions of the governing differential equations in terms of the source/sink, vertex viscosity and radii ratio parameters have been obtained. We have examined the impact of source/sink and radii ratio parameters on the velocity and microrotational velocity of a polar fluid by taking mixed type of thermal condition on the surface of inner cylinder. It is established that the gap between cylinders and source or sink parameter plays an important role in controlling the properties of fluid flow. The impacts of the source parameter on the velocity and microrotational velocity have increasing tendency while decreasing tendency is due to the sink. The influence of thermal boundary conditions on the inner cylinder strongly depends on the gap between the cylinders and this result can be used for modeling in such types of system. In this case, we obtain that the skin-friction of a polar fluid on outer cylinder decreases with heat source and increases with sink for isothermal and constant heat flux cases while reverse phenomenon occurs on inner cylinder.

\section{Acknowledgements}

The author is thankful to UGC, New Delhi, India for the Economical endorsement in the form of a Junior Research Fellowship to finish this work.

\section{References}

[1] Aung, W. (1972) Fully Developed Laminar Free Convection between Vertical Plates Heated Asymmetrically. International Journal of Heat and Mass Transfer, 15, 1577-1580. https://doi.org/10.1016/0017-9310(72)90012-9

[2] Nelson, D.J. and Wood, B.D. (1973) Combined Heat and Mass Transfer Natural Convection between Vertical Parallel Plates. International Journal of Heat and Mass Transfer, 32, 1779-1787. https://doi.org/10.1016/0017-9310(89)90059-8

[3] Aero, E.L., Bulygin, A.N. and Kuvshinski, E.V. (1965) Asymmetric Hydromechanics. Journal of Applied Mathematics and Mechanics, 29, 333-346. https://doi.org/10.1016/0021-8928(65)90035-3

[4] D’ep, N.V. (1968) Equations of a Fluid Boundary Layer with Couple Stresses. Journal of Applied Mathematics and Mechanics, 32, 777-778. https://doi.org/10.1016/0021-8928(68)90110-X

[5] Cowin, S.C. (1974) The Theory of Polar Fluids. Advances in Applied Mechanics, 14, 279-347. https://doi.org/10.1016/S0065-2156(08)70034-6 
[6] Chaturani, P. and Biswas, D. (1984) A Comparative Study of Poiseuille Flow of a Polar Fluid Under Various Boundary Conditions with Applications to Blood Flow. Rheologica Acta, 23, 435-445. https://doi.org/10.1007/BF01329196

[7] Singh, S.K., Jha, B.K. and Singh, A.K. (1997) Natural Convection in Vertical Concentric Annuli under a Radial Magnetic Field. Heat and Mass Transfer, 32, 399-401. https://doi.org/10.1007/s002310050137

[8] Raptis, A. and Takhar, H.S. (1999) Polar Fluid through a Porous Medium. Acta Mechanica, 135, 91-93. https://doi.org/10.1007/BF01179048

[9] Ogulu, A. (2005) On the Oscillating Plate Temperature Flow of a Polar Fluid past a Vertical Porous Plate in the Presence of Couple Stresses and Radiation. International Communications in Heat Mass Transfer, 32, 1231-1243. https://doi.org/10.1016/j.icheatmasstransfer.2005.05.002

[10] Cheng, C.Y. (2006) The Effect of Temperature-Dependent Viscosity on the Natural Convection Heat Transfer from a Horizontal Isothermal Cylinder of Elliptic Cross Section. International Communications in Heat and Mass Transfer, 33, 1021-1028. https://doi.org/10.1016/j.icheatmasstransfer.2006.02.019

[11] Cheng, C.Y. (2009) Natural Convection Heat Transfer from a Horizontal Isothermal Elliptical Cylinder with Internal Heat Generation. International Communications in Heat and Mass Transfer, 36, 346-350. https://doi.org/10.1016/j.icheatmasstransfer.2009.01.004

[12] Patil, P.M. (2008) Effects of Free Convection on the Oscillatory Flow of a Polar Fluid through a Porous Medium in the Presence of Variable Heat Flux. Journal of Engineering Physics and Thermo Physics, 81, 905-922. https://doi.org/10.1007/s10891-009-0122-Z

[13] Molla, M.M., Hossain, M.A. and Paul, M.C. (2006) Natural Convection Flow from an Isothermal Horizontal Circular Cylinder in Presence of Heat Generation. International Journal of Engineering Science, 44, 949-958. https://doi.org/10.1016/j.ijengsci.2006.05.002

[14] Patil, P.M. and Kulkarni, P.S. (2008) Effects of Chemical Reaction on Free Convective Flow of a Polar Fluid through a Porous Medium in the Presence of Internal Heat Generation. International Journal of Thermal Sciences, 47, 1043-1054. https://doi.org/10.1016/j.ijthermalsci.2007.07.013

[15] Ravi, S.K. and Singh, A.K. (2010) Natural Convection of a Polar Fluid in Vertical Concentric Annuli. International Journal of Applied Mathematics and Mechanics, 7, 70-81.

[16] Mohamed, R.A., Abo-Dahab, S.M. and Nofal, T.A. (2010) Thermal Radiation and MHD Effects on Free Convective Flow of a Polar Fluid through a Porous Medium in the Presence of Internal Heat Generation and Chemical Reaction. Mathematical Problems in Engineering, 2010, 27. https://doi.org/10.1155/2010/804719

[17] Ravi, S.K., Singh, A.K. and Alawadhi, K.A. (2011) Effect of Temperature Dependent Heat Source/Sink on Free Convective Flow of a Micropolar Fluid between Two Vertical Walls. International Journal of Energy and Technology, 3, 1-8.

[18] Perdikis, C. and Rapti, E. (2014) Magnetohydrodynamic Flow a Polar Fluid. International Journal of Mathematical Archive, 5, 7-11. 


\section{Nomenclature}

$a \quad$ Inner radius of the cylinder

$b \quad$ Outer radius of the cylinder

$S \quad$ Dimensionless source parameter

$\mathrm{Si} \quad$ Dimensionless sink parameter

$N^{\prime} \quad$ Angular viscosity

$N \quad$ Dimensionless angular velocity

$k \quad$ Vertex viscosity

$u^{\prime} \quad$ Stream wise velocity

$u \quad$ Dimensionless stream wise velocity

$r^{\prime} \quad$ Transverse coordinate

$r \quad$ Dimensionless transverse coordinate

$q^{\prime} \quad$ Constant heat flux

$q \quad$ Rate of heat flux

$T^{\prime} \quad$ Temperature of the fluid

$T \quad$ Dimensionless temperature

$T_{w}^{\prime} \quad$ Temperature of the wall

$T_{\infty}^{\prime} \quad$ Temperature of the free steam

$C_{p} \quad$ Specific heat at a constant pressure

$g \quad$ Acceleration due to gravity

$R \quad$ Vertex viscosity parameter

$j \quad$ Microinertia density

\section{Greek symbols}

$\rho \quad$ Density of fluid

$\beta \quad$ Coefficient of thermal expansion

$\lambda \quad$ Ratio of outer radius and inner radius, $b / a$

$\mu \quad$ Dynamic viscosity of the fluid

$\tau \quad$ Skin friction

$\tau_{1} \quad$ Skin friction at the wall $r=1$

$\tau_{\lambda} \quad$ Skin friction at the wall $r=\lambda$

$v \quad$ Kinematic viscosity of the fluid 
Appendix

$$
\begin{aligned}
& D_{0}=\frac{S}{2}\left(A+B \frac{\left(1-\lambda^{2}\right)}{2}\right)+C, \quad D_{1}=A-B \log [\lambda], \quad A_{1}=\frac{D_{0}}{D_{1}}, \\
& A_{2}=\frac{S \lambda^{2}}{4}-A_{1} \log [\lambda], \quad A_{3}=\frac{-2 R}{1+R}, \quad A_{4}=\frac{-A_{2}}{1+R}, \quad A_{5}=\frac{-A_{1}}{1+R}, \quad A_{6}=\frac{S}{4(1+R)}, \\
& A_{7}=\frac{A_{3}}{4}, \quad A_{8}=\frac{A_{4}-A_{5}}{4}, \quad A_{9}=\frac{A_{5}}{4}, \quad A_{10}=\frac{A_{6}}{16}, \quad A_{11}=A_{8}+A_{10}, \\
& A_{12}=2 A_{8}+A_{9}+4 A_{10}, \quad A_{13}=2\left(1+A_{7}\right), \quad A_{14}=A_{8} \lambda^{2}+A_{9} \lambda^{2} \log [\lambda]+A_{10} \lambda^{4}, \\
& A_{15}=A_{7} \lambda^{2}, \quad A_{16}=A_{15}-A_{13} \log [\lambda], \quad A_{17}=A_{12} \log [\lambda]-A_{14}, \\
& A_{18}=A_{16}+2 \lambda^{2} \log [\lambda], \quad A_{19}=\frac{A_{17}+A_{11}}{A_{18}-A_{7}}, \quad A_{20}=-A_{19} \lambda^{2}, \\
& A_{21}=-A_{11}-A_{7} \frac{A_{17}+A_{11}}{A_{18}-A_{7}}, \quad A_{22}=-\left(A_{12}+A_{19} A_{13}+2 A_{20}\right), \\
& q_{0}=C-\frac{S i}{4}\left(2 A+B\left(1-\lambda^{2}\right)\right), \quad q_{1}=A-B \log [\lambda], \quad q_{2}=\frac{q_{0}}{q_{1}}, \\
& q_{3}=-\left(q_{2} \log [\lambda]+\frac{S i \lambda^{2}}{4}\right), \quad q_{4}=\frac{-2 R}{1+R}, \quad q_{5}=\frac{-q_{3}}{1+R}, \quad q_{6}=\frac{-q_{2}}{1+R}, \\
& q_{7}=\frac{-S i}{4(1+R)}, \quad q_{8}=\frac{-q_{4}}{4}, \quad q_{9}=\frac{q_{5}-q_{6}}{4}, \quad q_{10}=\frac{q_{6}}{4}, \quad q_{11}=\frac{q_{7}}{16}, \quad q_{12}=q_{9}+q_{11}, \\
& q_{13}=2 q_{9}+q_{10}+4 q_{11}, \quad q_{14}=2\left(1+q_{8}\right), \quad q_{15}=q_{9} \lambda^{2}+q_{16} \lambda^{2} \log [\lambda]+q_{11} \lambda^{4}, \\
& q_{16}=q_{14}-2 \lambda^{2}, \quad q_{17}=q_{8} \lambda^{2}-q_{16} \log [\lambda], \quad q_{18}=q_{15}-q_{13} \log [\lambda], \quad q_{19}=\frac{q_{12}-q_{18}}{q_{17}-q_{8}}, \\
& q_{20}=-q_{19} \lambda^{2}, \quad q_{21}=-q_{18}-q_{19} q_{17}, \quad q_{22}=-\left(q_{13}+q_{16} q_{19}\right) .
\end{aligned}
$$

Submit or recommend next manuscript to SCIRP and we will provide best service for you:

Accepting pre-submission inquiries through Email, Facebook, LinkedIn, Twitter, etc. A wide selection of journals (inclusive of 9 subjects, more than 200 journals)

Providing 24-hour high-quality service

User-friendly online submission system

Fair and swift peer-review system

Efficient typesetting and proofreading procedure

Display of the result of downloads and visits, as well as the number of cited articles Maximum dissemination of your research work

Submit your manuscript at: http://papersubmission.scirp.org/

Or contact jamp@scirp.org 